\title{
Anabasine Measurement
}

National Cancer Institute

\section{Source}

National Cancer Institute. Anabasine Measurement. NCI Thesaurus. Code C147298.

The determination of the amount of anabasine present in a sample. 